Int. J. Contemp. Math. Sciences, Vol. 2, 2007, no. 31, 1527 - 1543

\title{
Coherent Systems on Singular Genus One Curves
}

\author{
E. Ballico ${ }^{1}$ and F. Prantil ${ }^{2}$ \\ Dept. of Mathematics \\ University of Trento \\ 38050 Povo (TN), Italy \\ ballico@science.unitn.it \\ prantil@science.unitn.it
}

\begin{abstract}
Here we prove the existence of $\alpha$-stable coherent systems on a singular genus 1 curve. This paper is a continuation of [2].

\section{Mathematics Subject Classification: 14H60}

Keywords: Coherent systems, vector bundles, torsion free sheaves, elementary transformations
\end{abstract}

\section{INTRODUCTION}

A coherent system on a curve $C$ is a pair $(E, V)$ where $E$ is a torsion free sheaf and a subspace $V \subset H^{0}(E)$. A coherent subsystem is a pair $(F, W)$ where $F$ is a subsheaf of $E$ and $W \subset V \cap H^{0}(F)$. Fix a real number $\alpha$ and call $\mu_{\alpha}(E, V)=\frac{\operatorname{deg}(E)+\alpha \operatorname{dim}(V)}{\operatorname{rk}(E)}$ the $\alpha$-slope of $(E, V)$. Then a coherent system $(E, V)$ is called stable (resp. semistable) if $\mu_{\alpha}(E, V)<\mu_{\alpha}(F, W)$ (resp. $\left.\mu_{\alpha}(E, V) \leq \mu_{\alpha}(F, W)\right)$ for every coherent subsystem $(F, W)$. With this definition the moduli spaces of stable coherent systems were built (see [10]). For the background theory of coherent systems see [10] and [5]. The case of smooth genus 1 curves (i.e. elliptic curves) is investigate in [11]. In this paper we will show the existence of $\alpha$-stable coherent systems on a singular genus 1 curve. This work is a continuation of a previous paper [2].

In section 2 we give the basic proprieties of torsion free sheaves and we study the elementary transformation of a polystable torsion free sheaf with pairwise non-isomorphic factors.

In section 3 we investigate the evaluation map of a polystable torsion free sheaf and apply the results to give examples of $\alpha$-stable coherent systems when $\operatorname{dim}(V)<\operatorname{rk}(E)$.

\footnotetext{
${ }^{1}$ The author was partially supported by MIUR and GNSAGA of INdAM (Italy).

${ }^{2}$ The author was partially supported by MIUR.
} 
In section 4 we conclude giving some examples of $\alpha$-stable coherent systems in other cases, namely the cases $\operatorname{dim}(V)=\operatorname{rk}(E), \operatorname{dim}(V)=\operatorname{rk}(E)+1$ and $\operatorname{dim}(V)=\operatorname{rk}(E)+2$.

\section{BASIC PROPRIETIES OF TORSION FREE SHEAVES}

Let $C$ be an integral projective curve such that $p_{a}(C)=1$. Assume $C$ singular and call $Q$ the unique singular point of $C$. Hence $C$ has either an ordinary cusp or an ordinary node, i.e. either an $A_{2}$-singularity or an $A_{1^{-}}$ singularity. Let $m_{Q}$ denote the maximal ideal of the local ring $\mathcal{O}_{C, Q}$.

Notation 1. Fix an integer $n \geq 1$, a rank $n$ torsion free $\mathcal{O}_{C, Q}$-module $M$ and a torsion free coherent sheaf $F$ on $C$. There is a unique integer $a$ such that $0 \leq a \leq n$ and $M \cong \mathcal{O}_{C, Q}^{\oplus(n-b)} \oplus m_{Q}^{\oplus b}$ ([7] or [6], Remark 4 at p.25). We will say that $M$ has type $b$. We will say that $F$ has local type $b$ if the germ of $F$ at $Q$ has type $b$.

Remark 1. Fix integers $r, d$ such that $r>0$ and $(r, d)=1$. There is a unique, up to isomorphism, non-locally free but torsion free sheaf $F_{r, d}$ on $C$ with rank $r$ and degree $d$. This was proved in [8] in the rank 1 case, in [12, Corollary 4.6] for any rank on a nodal curve and in [4, Theorem 22] on a cuspidal curve. We will give a different and simpler proof in Theorem 1 .

Remark 2. Notice that $E$ and $E^{*}$ have the same type.

Lemma 1. Let $E$ be a torsion free semistable sheaf on $C$. Then $E^{*}$ is semistable.

Proof. Assume that $E^{*}$ is not semistable and take a destabilizing sequence

$$
0 \rightarrow A \rightarrow E^{*} \rightarrow B \rightarrow 0
$$

with $A, B$ torsion free and $\mu(A)>\mu(B)$. Since $C$ is Gorenstein, the natural map $E \rightarrow E^{* *}$ is an isomorphism. For any torsion free sheaf $F$ on $C$ we have $\operatorname{Ext}^{i}\left(F, \omega_{C}\right)=0$ for every integer $i \geq 1$ ([6], Lemma 2.5.3). Hence dualizing (1) we get an exact sequence

$$
0 \rightarrow B^{*} \rightarrow E \rightarrow A^{*} \rightarrow 0
$$

We get a contradiction to the semistability of $C$, because $\operatorname{deg}\left(F^{*}\right)=-\operatorname{deg}(F)$ for any torsion free sheaf on $C$ ([6], Prop. 3.1.6, part 2); here we use that $C$ is Gorenstein.

Lemma 2. Fix $P \in C_{\text {reg }}$ and integers $n \geq 2, n-1 \leq a \leq n$ and $0<d<n$. Take a pairwise non-isomorphic $L_{i} \in \operatorname{Pic}^{0}(C), 1 \leq i \leq a$, and (if $a \neq n$ ) set $L_{n}:=F_{1,0}$. Set $F:=\oplus_{i=1}^{n} L_{i}$. Let $E$ be a sheaf obtained from $F$ making $d$ general positive elementary transformations supported by $P$. Then $E$ is a semistable torsion free sheaf with degree $d$, rank $n$ and type $n-a$. 
Proof. $E$ has type $n-a$, rank $n$ and degree $d$, because $P \in C_{\text {reg }}$. Assume that the statement of the lemma is not true and take an exact sequence on $C$ :

$$
0 \rightarrow A \rightarrow E \rightarrow B \rightarrow 0
$$

with $\mu(A)>\mu(E)$ and $B$ torsion free. Set $r:=\operatorname{rank}(A)$. Hence $0<r<n$. Up to a twist by a degree 0 line bundle we may assume that no direct factor of $F$ is trivial. This assumption implies $h^{0}(C, F)=0$. Hence $h^{1}(C, F)=0$ (RiemannRoch). Since $F$ is a subsheaf of $E$ and $E / F$ is torsion, we get $h^{1}(C, E)=0$, i.e. $h^{0}(C, E)=d$ (Riemann-Roch). Seen $F$ as a subsheaf of $E$ we easily see that $\operatorname{rank}(A \cap F)=\operatorname{rank}(A)$ and $\operatorname{deg}(A)-d \leq \operatorname{deg}(F \cap A) \leq \operatorname{deg}(A)$. First assume $d=1$. Since $h^{0}(C, E)=1$ and $\operatorname{deg}(A)>0$, we get $\operatorname{deg}(A)=1$ and $\operatorname{deg}(B)=0$. The generically surjective map $F \rightarrow B$ and the polystability of $F$ implies that $B$ is a direct factor of $F$. Since the indecomposable factors are pairwise non-isomorphic, $F$ has only finitely many direct factors: choose a non-empty finite subset $S$ of the set $\{1, \ldots, n\}$ and take $\bigoplus_{i \in S} L_{i}$. Since $E$ is obtained from $F$ making a general positive elementary transformation supported by $P$, each $\oplus_{i \in S} L_{i}$ is saturated in $E$ if $\sharp(S)<n$, contradiction. The case $d=1$ conclude the case $n=2$. Hence we may assume $n \geq 3, d \geq 2$ and use induction on $n$. For the fixed rank $n$ we use induction on the degree $d$, i.e. we assume that the result is true for all pairs $\left(n^{\prime}, d^{\prime}\right)$ such that either $1 \leq d^{\prime}<n^{\prime}<n$ or $n^{\prime}=n$ and $1 \leq d^{\prime} \leq d-1$. Set $M:=\oplus_{i=2}^{n} L_{i}$ and let $N$ be a sheaf obtained from $M$ making $d-1$ general positive elementary transformations supported by $P$. Since $d-1<n-1$, the inductive assumption implies the semistability of $N$. By the openness of semistability it is sufficient to show the existence of a semistable sheaf obtained from $F$ making $d$ positive elementary transformations supported by $P$. Hence it is sufficient to prove the existence of a semistable sheaf $G$ obtained from $L_{1} \oplus N$ making a positive elementary transformation supported by $P$. Take any sheaf $G$ obtained from $L_{1} \oplus N$ making a positive elementary transformation supported by $P$. $G$ fits in an exact sequence

$$
0 \rightarrow N \rightarrow G \rightarrow L_{1}(P) \rightarrow 0
$$

Since $a>0, L_{1}(P)$ is a degree 1 line bundle. Take any exact sequence (4). There is a sheaf $G^{\prime}$ obtained from $G$ making a negative elementary transformation supported by $P$ and fitting in an exact sequence

$$
0 \rightarrow N \rightarrow G^{\prime} \rightarrow L_{1} \rightarrow 0
$$

Since $L_{1}$ is locally free, $\operatorname{Ext}^{1}\left(L_{1}, N\right)=0$. Since $L_{1}$ is locally free and $N$ is semistable of degree $d-1>0, \operatorname{Hom}\left(L_{1}, N\right)$ is semistable and of degree $d-1>0$. The duality for locally Cohen-Macaulay schemes and the triviality of $\omega_{C}$ gives $h^{1}\left(C, \operatorname{Hom}\left(L_{1}, N\right)\right)=h^{0}\left(C, \operatorname{Hom}\left(L_{1}, N\right)^{*}\right)$. Lemma 1 implies the semistability of $\operatorname{Hom}\left(L_{1}, N\right)^{*}$. Hence $h^{0}\left(C, \operatorname{Hom}\left(L_{1}, N\right)^{*}\right)=0$. The local-toglobal spectral sequence for the global Ext ${ }^{1}$ functor gives $\operatorname{Ext}^{1}\left(L_{1}, N\right)=0$. Thus the extension (5) splits. We just checked that every sheaf $G$ fitting in (4) 
may be obtained from $L_{1} \oplus N$ making a positive elementary transformation supported by $P$. Take a general $G$ fitting in an exact sequence (4) and assume that $G$ is not semistable. Fix an exact sequence on $C$ :

$$
0 \rightarrow D \rightarrow G \rightarrow M \rightarrow 0
$$

with $\mu(D)>d / n, M$ torsion free and $D$ with maximal slope. Hence $D$ is semistable. Set $s:=\operatorname{rank}(D)$ and $D^{\prime}:=L_{1} \oplus D \cap M$. Let $\pi: G \rightarrow L_{1}(P)$ the surjective map in (4). First assume $D \subseteq N$. Since $N$ is semistable, $\mu(D) \leq \mu(N)$. Since $\mu(G)>\mu(N)$, we get a contradiction. Thus $\pi(D) \neq 0$. First assume $\pi(D) \neq L_{1}(P)$, i.e. $\operatorname{deg}(\pi(D)) \leq 0$. If $s=1$, we have $D \cong \pi(D)$ and hence $\operatorname{deg}(D) \leq 0$, contradiction. Hence $s \geq 2$ and the sheaf $D \cap N$ has rank $s-1$. Since $N$ is semistable, we get $\operatorname{deg}(D)=\operatorname{deg}(D \cap N)+\operatorname{deg}(\pi(D)) \leq$ $(s-1)(d-1) /(n-1)<s d / n$ (since $d<n)$, contradiction. Now assume $\pi(D)=L_{1}(P)$. If $s=1$ this implies that (5) splits, contradicting the existence of a non-trivial extension (5), because $\mu(N)<\mu\left(L_{1}(P)\right)$. Hence we may assume $s \geq 2$. We have an exact sequence

$$
0 \rightarrow N \cap D \rightarrow D \rightarrow L_{1}(P) \rightarrow 0
$$

with $\mu_{+}(N \cap D) \leq(d-1) /(n-1)$. The proof just given (which uses the local freeness of $\left.L_{1}(P)\right)$ and the semistability of $N$ gives $D \cong(D \cap N) \oplus L_{1}(P)$. Since $D \subset G$, again we get that (5) splits, contradiction.

Corollary 1. Fix integers $n, d, a$ such that $n>0$ and $n-1 \leq a \leq n$. If $a=n$ let $F$ be a general element of $\operatorname{Pic}^{0}(Y)^{\oplus n}$. If $a=n-1$ let $F$ be the direct sum of $F_{1,0}$ and a general element of $\operatorname{Pic}^{0}(Y)^{\oplus(n-1)}$. There is a polystable torsion free sheaf $E$ on $C$ such that $\operatorname{rank}(E)=n, \operatorname{deg}(E)=d, E$ has type $(a, n-a)$, the indecomposable factors of $E$ are pairwise non-isomorphic and $E$ is obtained from $F$ making d positive elementary transformations..

Proof. Twisting with a line bundle we reduce to the case $0 \leq d \leq n-1$. If $d=0$, then use a direct sum of $a$ pairwise non-isomorphic line bundles of degree 0 and (if $a \neq n$ ) the unique rank 1 and degree 0 torsion free sheaves. There are infinitely many such sheaves, because $\operatorname{dim}\left(\operatorname{Pic}^{0}(C)\right)=1$ and we may twist (if necessary) all the locally free factors by general $\left(L_{1}, \ldots, L_{n}\right) \in \operatorname{Pic}^{0}(C)^{\oplus n}$. If $(n, d)=1$, then use Lemma 2 and that if $(n, d)=1$ stability and semistabily are the same. Now assume $(n, d)>1$. Set $n^{\prime}:=n /(n, d)$ and $d^{\prime}:=d /(n, d)$. Fix integers $a_{i}, 1 \leq i \leq(n, d)$, such that $n^{\prime} \leq a_{i} \leq n^{\prime}$ and $\sum_{i=1}^{(n, d)} a_{i}=a$. Hence $a_{i}=n^{\prime}$, except at most for one index $i$. Since $\left(n^{\prime}, d^{\prime}\right)=1$, we just proved the existence of stable torsion free sheaves $F_{i}$ such that $F_{i}$ has degree $d^{\prime}$ and type $a_{i}, n^{\prime}-a_{i}$. Take a general $\left(L_{1}, \ldots, L_{(n, d)}\right) \in \operatorname{Pic}^{0}(C)^{\oplus(n, d)}$ and set $E:=\oplus_{i=1}^{(n, d)} F_{i} \otimes L_{i}$.

Theorem 1. Fix integers $r, d$ such that such that $r>0(r, d)=1$. Let $L$ be a line bundles of degree $d$. Then:

(i) there exists a unique stable vector bundle of rank $r$ and determinant $L$; 
(ii) there exists a unique stable torsion free sheaf of rank $r$, degree $d$; it has type 1 ;

(iii) if $r \geq 2$ every stable torsion free sheaf $E$ with degree $d$ and rank $r$ can be written in a sequence

$$
0 \longrightarrow E_{1} \longrightarrow E \longrightarrow E_{2} \longrightarrow 0
$$

where $E_{1}$ is a stable locally free sheaf and $E_{2}$ is a stable torsion free sheaf such that $\operatorname{rank}\left(E_{1}\right) \operatorname{deg}(E)-\operatorname{rank}(E) \operatorname{deg}\left(E_{1}\right)=1$.

Proof. The locally free case is [14], Th. 5.1. We extend his proof to the not locally free case. Let $r_{1}, d_{1}$ the the unique pair of integers with $r_{1} d-r d_{1}=1$ and $0<r_{1}<r$. Set $d_{2}:=d-d_{1}$ and $r_{2}:=r-r_{1}$. Fix a stable vector bundle $E_{1}$ of degree $d_{1}$ and rank $r_{1}$. Let $E_{2}$ be the unique stable torsion free sheaf of degree $d_{2}$ and rank $r_{2}$ which is not locally free. Since $E_{1}$ is locally free we have $\operatorname{dim}\left(\operatorname{Ext}^{1}\left(E_{2}, E_{1}\right)=1\right.$. Consider a non trivial extension

$$
0 \rightarrow E_{1} \rightarrow E \rightarrow E_{2} \rightarrow 0 .
$$

As the locally free case ([14], Th. 5.1) we see that $E$ is stable and $\operatorname{dim}\left(\operatorname{Hom}\left(E_{1}, E\right)\right)=1$. It is clear that $E$ is not locally free and unique.

Notation 2. For all integers $n>0$ and $d$ let $U(n, d)$ denote the set of all polystable vector bundles $E$ on $C$ such that $\operatorname{rank}(E)=n, \operatorname{deg}(E)=d$ and the indecomposable factors $E$ are pairwise non-isomorphic. Let $V(n, d)$ denote the set of all polystable torsion free sheaves $E$ on $C$ such that $\operatorname{rank}(E)=n$, $\operatorname{deg}(E)=d$, the indecomposable factors $E$ are pairwise non-isomorphic and $E$ is not locally free. Fix an integer $k>0$. For all $\alpha \in \mathbb{R}, \alpha>0$, let $U(\alpha ; n, d, k)$ (resp. $V(\alpha ; n, d, k))$ denote the set of all $\alpha$-stable coherent systems $(E, V)$ such that $E \in U(n, d)$ (resp. $E \in V(n, d))$.

Remark 3. Fix integers $n>0, k>0$ and $d$. $U(n, d)$ is a non-empty irreducible variety of dimension $(n, d) . V(n, d)$ is a non-empty irreducible variety of dimension $(n, d)-1$. Notice that each $F \in V(n, d)$ has type 1. The main difference with respect to the locally free case is that for any stable torsion free sheaf $G$ non locally free we have $G \otimes L \cong G$ for all $L \in \operatorname{Pic}(C)$. Now assume $d \geq k$. Notice that $h^{0}(C, E)=d$ for all $E \in$ $U(n, d) \cup V(n, d)$. Assume $U(\alpha ; n, d, k) \neq \emptyset(\operatorname{resp} . V(\alpha ; n, d, k) \neq \emptyset)$ and take $(E, V) \in U(\alpha ; n, d, k)$ (resp. $(E, V) \in V(\alpha ; n, d, k))$. Since $\alpha$-stability is an open condition, $(E, W)$ is $\alpha$-stable for a general $k$-dimensional linear subspace $W$ of $H^{0}(C, E)$. Similarly, the same is true for a general $E^{\prime} \in U(n, d)$ (resp. $\left.E^{\prime} \in V(n, d)\right)$. Hence if $U(\alpha ; n, d, k) \neq \emptyset(\operatorname{resp} . V(\alpha ; n, d, k) \neq \emptyset)$, then $U(\alpha ; n, d, k)$ (resp. $V(\alpha ; n, d, k))$ is irreducible and of dimension $(n, d)+k(d-k)$ (resp. $(n, d)-1+k(d-k))$.

Remark 4. Let $Y$ be an integral projective curve. Set $g:=p_{a}(Y)$. Let $E$ be a vector bundle. If $g \geq 2$, then $E$ is a limit of a flat family of stable vector bundles ([3], Lemma 2.4, and [9], Prop. 2.1 and Cor. 2.2). If $g=1$, then the proof of [9], Prop. 2.1 and Cor. 2.2, gives that $E$ is a limit of a flat family 
of semistable vector bundles; indeed, it is sufficient to use that semistability is an open condition.

Lemma 3. Fix integers $r$, a such that $r>0$ and any $F \in V(r, a)$. Let $\mathbb{K}_{Q}$ denote the skyscraper sheaf on $C$ supported by $Q$ and with $h^{0}\left(C, \mathbb{K}_{Q}\right)=1$. Let $u: F \rightarrow \mathbb{K}_{Q}$ be a general surjection. Then $E:=\operatorname{Ker}(u)$ is a semistable vector bundle.

Proof. Since $F$ has local type 1, either $E$ is locally free or it has local type 2. Since $\mathbb{K}_{Q}$ has finite support, the restriction map $H^{0}\left(C, \operatorname{Hom}\left(F, \mathbb{K}_{Q}\right)\right) \rightarrow$ $\operatorname{Hom}(F \mid\{Q\}, \mathbb{K})$ is bijective. Hence $E$ is locally free for general $u$. Notice that $H^{0}\left(C, \operatorname{Hom}\left(F, \mathbb{K}_{Q}\right)\right) \cong \oplus_{i} H^{0}\left(C, \operatorname{Hom}\left(F_{i}, \mathbb{K}_{Q}\right)\right)$, where $\oplus_{i} F_{i}$ is a decomposition of $F$ into irreducible factors. Since $E$ has only finitely many direct factors, the generality of $u$ shows that no $F_{i}$ is a factor of $E$. Hence each indecomposable factor of $E$ has slope $<a / r$. Hence $h^{0}(C, \operatorname{Hom}(E, F))=r$. Notice that $h^{0}(C, \operatorname{Hom}(M, F))=r$ for every semistable vector bundle on $C$ with degree $a-1$ and rank $r$. Take a flat family $\left\{E_{t}\right\}_{t \in T}$ of vector bundles on $C$ parametrized by an integral curve $T$ such that there is $o \in T$ such that $E_{o} \cong E$ and $E_{t}$ is semistable for all $t \in T \backslash\{o\}$ (Remark 4). We saw that the integer $h^{0}\left(C, \operatorname{Hom}\left(E_{t}, F\right)\right), t \in T$, does not depend from $t \in T$. Hence these Hom-cohomology groups fit together to form a rank $r$ vector bundle on $T$. In particular their total space is irreducible. Since there is $E_{o} \rightarrow F$ which is injective, there an inclusion $j_{t}: E_{t} \rightarrow F$ for general $t$. Since $E_{t}$ is locally free, while $F$ is not locally free, $E_{t}$ and $F$ have the same rank and $\operatorname{deg}\left(E_{t}\right)=\operatorname{deg}(F)-1$, the injectivity of $j_{t}$ implies $\operatorname{Coker}\left(j_{t}\right) \cong \mathbb{K}_{Q}$. Since $u$ is general and semistability is an open condition, $E$ is semistable.

Lemma 4. Fix any $E \in U(r, d)$ and let $G$ be a general extension of $\mathbb{K}_{Q}$ by $E$. Then $G$ is torsion free of type 1 and semistable.

Proof. A general extension of $\mathbb{K}_{Q}$ by $E$ is torsion free. Any torsion free extension of $\mathbb{K}_{Q}$ by $E$ has type 1 . Since $C$ is Gorenstein, any torsion free sheaf $A$ on $C$ is reflexive, i.e. the natural map $A \rightarrow A^{* *}$ is an isomorphism. Hence $G \cong G^{* *}$. By Lemma 1 it is sufficient to prove that $G^{*}$ is semistable. By Lemma 1 it is sufficient to prove that $G^{*}$ is semistable. Fix a general extension

$$
0 \rightarrow E \rightarrow G \rightarrow \mathbb{K}_{Q} \rightarrow 0
$$

Since $G^{*}$ is torsion free, dualizing $(9)$ we get an exact sequence

$$
0 \rightarrow G^{*} \rightarrow E^{*} \rightarrow \mathbb{K}_{Q} \rightarrow 0
$$

Consider the general surjection $u: E^{*} \rightarrow \mathbb{K}_{Q}$. Lemma 3 gives the semistability of the vector bundle $\operatorname{Ker}(u)$ is semistable. Hence to prove the lemma it is sufficient to note that any surjection $u$ gives an exact sequence (10) (for some $G^{*}$ of type 1) and that dualizing it we get an exact sequence (9) (again, because the torsion freeness of $E=E^{* *}$ implies that the associated map $E^{* *} \rightarrow G^{* *}$ is injective). 
Lemma 5. Fix integers $r$, $d$ such that $r>0$. Then there exist $E \in U(r, d)$, $F \in U(r, d+1)$ and an injective map $j: E \rightarrow F$. We may take as $E$ (resp. $F)$ a general element of $U(r, d)$ (resp. $U(r, d+1))$.

Proof. Write $d=c r+e$ with $c, e \in \mathbb{Z}$ and $0 \leq e \leq r-1$. If $e=0$, then this is Corollary 1 for one positive elementary transformation and $a=n$ (i.e. taking $M:=\oplus_{i=1}^{r} L_{i}$ with $L_{i} \in \operatorname{Pic}^{c}(C), L_{i} \not L_{j}$ for all $i \neq j$, as $E$. If $0<e \leq r-2$, then $E$ is obtained from $M$ making $e$ general positive elementary transformation, while $G$ is obtained from $E$ making a further general positive elementary transformation. Now assume $e=r-1$. Set $G:=\oplus_{i=1}^{r} R_{i}$ with $R_{i} \in \operatorname{Pic}^{c+1}(C), R_{i} ¥ R_{j}$ for all $i \neq j . \quad E$ is obtained from $G$ making a general negative elementary transformation. Apply the dual of Lemma 2 or of Corollary 1 to get that $E \in U(r, d)$. The last statement follows from the proof and the openness of semistability.

Lemma 6. Fix integers $r, d$ such that $r>0$. Then there exist $E \in V(r, d)$, $F \in V(r, d+1)$ and an injective map $j: E \rightarrow F$. We may take as $E$ a general element of $V(r, d)$.

Proof. Copy the proof of Lemma 5, just quoting the case $a=n-1$ instead of the case $a=n$ of Lemma 2 and Corollary 1. The last statement follows from the proof and the openness of semistability.

Iterating $a$ times Lemmas 5 and 6 we get the following results.

Lemma 7. Fix integers $r, d, a$ such that $r>0$ and $a>0$. Then there exist $E \in U(r, d), F \in U(r, d+a)$ and an injective map $j: E \rightarrow F$. We may take as $E$ (resp. F) a general element of $U(r, d)$ (resp. $U(r, d+a))$.

Lemma 8. Fix integers $r, d, a$ such that $r>0$ and $a>0$. Then there exist $E \in V(r, d), F \in V(r, d+a)$ and an injective map $j: E \rightarrow F$. We may take as $E($ resp. F) a general element of $V(r, d)($ resp. $V(r, d+a))$.

Proposition 1. Fix integers $r, d$ such that $r>0$. Then there exist $E \in$ $U(r, d), G \in V(r, d+1)$ and an injective map $j: E \rightarrow G$. We may take as $E$ a general element of $U(r, d)$ or as $G$ a general element of $V(r, d+1)$.

Proof. Take any triple $(A, B, m)$ with $A$ locally free, $B$ of type $1, u: A \rightarrow B$ injective, $\operatorname{rank}(A)=\operatorname{rank}(B)$ and $\operatorname{deg}(B)=\operatorname{deg}(A)-1$. Any such triple $(A, B, m)$ induces a non-split exact sequence

$$
0 \rightarrow A \rightarrow B \rightarrow \mathbb{K}_{Q} \rightarrow 0
$$

Conversely, any triple $(A, B, m)$ as above gives a non-split exact sequence (11). Fix $P \in C_{\text {reg }}$ and take any sheaf $B^{\prime}$ obtained from $B$ making a positive elementary transformation support by $P$. Hence there is an inclusion $m^{\prime}$ : $B \rightarrow B^{\prime}$. The saturation $A^{\prime}$ of $m^{\prime} \circ m(A)$ in $B^{\prime}$ is a vector bundle obtained from $A$ making a positive elementary transformation supported by $P$. By construction there in an injection $A^{\prime} \rightarrow B^{\prime}$. Now we want to show that every 
positive elementary transformation $\tau$ supported by $P$ arises in the previous way. $\tau$ from a negative elementary transformation of $A^{*}$, i.e. from a surjection $u: A^{*} \rightarrow \mathbb{K}_{P}$, just dualizing $\operatorname{Ker}(u)$. Since $P \neq Q$, the inclusion $m$ induces an isomorphism $B^{*}\left|\{P\} \rightarrow A^{*}\right|\{P\}$. Thus $u$ induces a negative elementary transformation of $B^{*}$ supported by $P$, i.e. a positive elementary transformation supported by $P$. Write $d=c r+e$ with $a, e \in \mathbb{Z}$ and $0 \leq e \leq r-1$. Since $(r, 1)=0$, the case $e=0$ is true by Lemma 4 . We fix a solution $E^{\prime} \in$ $U(r, c r)$ and $G^{\prime} \in V(a r, 1)$. Notice that $E^{\prime}$ is a direct sum of $r$ pair-wise non-isomorphic line bundles with the same degree, $c$. Now assume $e>0$. Let $E$ be a general vector bundle obtained from $E^{\prime}$ making $e$ general positive elementary transformations. Corollary 1 gives $E \in V(r, d)$. Let $G$ be the torsion free sheaf of type 1 obtained from $G^{\prime}$ making the $e$ positive elementary transformations induced (as in the first part of the proof) from the $e$ positive elementary transformations made to obtain $E$ from $E^{\prime}$. In the first part of the proof we saw that the inclusion $E^{\prime} \rightarrow G^{\prime}$ induces an inclusion $j: E \rightarrow G$. Since $G^{\prime}$ is a general extension of $\mathbb{K}_{Q}$ by $E^{\prime}, G$ is a general extension of $\mathbb{K}_{Q}$ by $E$. Lemma 4 gives the semistability of $G$. To conclude the proof it is sufficient to prove that a general sheaf obtained from $E^{\prime}$ making first an extension by $\mathbb{K}_{Q}$ and the $e$ positive elementary transformations is an element of $V(r, d+1)$. we know that it is semistable. Hence we may assume $h:=(r, d+1)>0$. We use induction on $r$, the case $r=1$ being obvious. By semicontinuity it is sufficient to prove the existence of $Z \in V(r, d+1)$ which is a torsion free extension of $E^{\prime}$ by $\mathbb{K}_{Q}$ and $e$ extensions $\mathbb{K}_{P_{i}}, 1 \leq i \leq e, P_{i} \in C_{\text {reg }}$ for all $i$. Write $E^{\prime}:=\oplus_{i=1}^{r} L_{i}, L_{i} \in \operatorname{Pic}^{c}(C)$ for all $i$, and $E_{j}:=\bigoplus_{i=1+(j-1) h}^{j h} L_{i}$. Hence $E^{\prime}=\oplus_{i=1}^{h} E_{i}$. Apply to each $E_{i}, 1 \leq i \leq h-1,(e+1) / h$ general positive elementary transformation to get $G_{i} \in U(r / h,(a r+e+1) / h)$ (Corollary 1$)$. Take as $G_{h}$ the sheaf obtained applying $(e+1) / h-1$ general positive elementary extensions to a general extension of $\mathbb{K}_{Q}$ by $E_{h} . E_{h} \in V(a r / h,(a r+e+1) / h)$ by the inductive assumption. Set $Z:=\bigoplus_{i=1}^{h} G_{i}$. Taking general the first $(h-1)(e+1) / h$ positive elementary transformations we may assume that $\operatorname{det}\left(G_{i}\right) \varsubsetneqq \operatorname{det}\left(G_{j}\right)$ for all $1 \leq i<j \leq h-1$. Hence $Z \in V(r, d+1)$. The last statement follows from the proof and the openness of semistability.

\section{Coherent systems With $k<n$}

Remark 5. Fix integers $d \geq n>0$ and a semistable torsion free sheaf $E$ on $C$ with rank $n$ and degree $d$. Hence $h^{0}(C, E)=d$ and $h^{1}(C, E)=0$. Fix any integer $m$ such that $0<m \leq n$ and take a general $m$-dimensional linear subspace $V$ of $H^{0}(C, E)$. It is easy to check that the evaluation map $e_{E, V}$ is injective.

Lemma 9. Fix integer $d>n>0$. Let $E$ be a semistable torsion free sheaf on $C$ with degree $d$ and rank $n$. Then $H^{0}(C, E)$ spans $E$ at each point of $C_{\text {reg }}$.

Proof. Fix $P \in C_{\text {reg. }}$. Hence $E(-P) \in V(n, d-n)$. Thus $h^{1}(C, E(-P))=0$. Thus $H^{0}(C, E)$ spans $E$ at each point of $C_{\text {reg }}$. 
Lemma 10. For every integer $n \geq 1, F_{n, n+1}$ is spanned.

Proof. Assume that the result is not true and call $A \varsubsetneqq F_{n, n+1}$ the image of the evaluation map. We have $h^{0}\left(X, F_{n, n+1}\right)=n+1$. Lemma 9 gives that $F_{n, n+1}$ is spanned outside $Q$. Hence $h^{0}(X, A)=n+1, \operatorname{rank}(A)=n$ and $\operatorname{deg}(A) \leq n$. Set $a:=\operatorname{deg}(A)$. Since $h^{0}(C, A)=n+1$, Riemann-Roch and Serre duality give $h^{0}\left(C, \operatorname{Hom}\left(A, \mathcal{O}_{X}\right)\right)=n+1-a>0$. Since $\operatorname{rank}(A) \leq n$ and $A$ is spanned, we get $a \geq 1$ and $A \cong \mathcal{O}_{C}^{\oplus(n+1-a)} \oplus B$ with $h^{0}(X, B)=a, \operatorname{deg}(B)=a, h^{1}(C, B)=0$ and $\operatorname{rank}(B)=a-1$. Hence if $a \geq 2$, then $\mu(B)=a /(a-1)>\mu\left(F_{n, n+1}\right)$, contradicting the stability of $F_{n, n+1}$. Now assume $a=1$, i.e. $A \cong \mathcal{O}_{C}^{\oplus n}$. Since $h^{0}(C, A)=n+1$, we get a contradiction.

Since every $E \in U(n, n+1)$ is stable, the same proof gives the following result.

Lemma 11. For every integer $n \geq 1$ every $E \in U(n, n+1)$ is spanned.

Lemma 12. Fix integers $d>n>0$. There are $E \in U(n, d)$ and $F \in V(n, d)$ such that $E$ and $F$ are spanned. For a general $(n+1)$-dimensional linear subspace $V \subseteq H^{0}(C, V)$ (resp. $W \subseteq H^{0}(C, F)$ ) the map evaluation map $e_{E, V}$ (resp. $e_{F, W}$ is surjective.

Proof. Lemma 9 shows that all elements of $U(n, d) \cup V(n, d)$ are spanned at each point of $C_{r e g}$. Now we will show the existence $E$ is spanned at $Q$. $E$ has local type 0 or 1 . If $d=n+1$, then all elements of $U(n, n+1) \cup V(n, n+1)$ are spanned at $Q$ (Lemmas 11 and 10). Assume $d \geq n+2$ and that the results is true for the integer $d^{\prime}:=d-1$. Take general $E^{\prime} \in U(n, d-1)$ and $F^{\prime} \in U(n, d-1)$. The proofs of Lemmas 5 and 6$)$ show the existence of inclusions $\left.j: E^{\prime} \rightarrow E, i: F^{\prime} \rightarrow F\right)$ such that $E \in U(n, d), F \in V(n, d)$, and $Q$ is neither in the support of $\operatorname{Coker}(j)$ nor in the support of $\operatorname{Coker}(i)$. Since $E^{\prime}$ and $F^{\prime}$ are spanned at $Q$, the existence of the maps $i, j$ gives that $E$ and $F$ are spanned at $Q$. Since spannedness is an open conditions in flat families of sheaf with constant cohomology, the general members of $U(n, d)$ and of $V(n, d)$ are spanned. The last part of the lemma is straightforward.

Lemma 13. Fix integers $d>n>0$ and $E \in U(n, d) \cup V(n, d)$. Then $E$ is spanned. For a general $(n+1)$-dimensional linear subspace $V \subseteq H^{0}(C, V)$ the map evaluation map $e_{E, V}$ is surjective.

Proof. Lemma 9 gives that $E$ is spanned at each point of $C_{r e g}$. Assume that

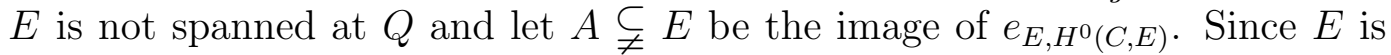
spanned at each point of $C_{r e g}, A$ has rank $n$ and the inclusion $j: A \rightarrow E$ has cokernel supported by $Q$. Set $a:=\operatorname{deg}(A)$. Hence $a<d$. Since $h^{0}(C, A)=$ $h^{0}(C, E)=d$, we have $h^{1}(C, E)=d-a$, i.e. $h^{0}\left(C, \operatorname{Hom}\left(E, \mathcal{O}_{C}\right)\right)=d-a$. Since $A$ is spanned, the last equality implies $A \cong \mathcal{O}_{C}^{\oplus(d-a)} \oplus B$ for some torsion free sheaf $B$ with degree $a$, rank $n-d+a$ and $h^{1}(C, B)=0$. Since $a>0$, $B \neq 0$, i.e. $n-d+a>0$. Since $0<a<d$ and $d>n, a /(n-d+a)>d / n$, 
i.e. $\mu(B)>\mu(E)$, contradicting the semistability of $E$. The last part is straightforward, because $E$ has local type 0 or 1 at $Q$.

Lemma 14. Fix integers $d \geq 2 n>0$. Let $E$ be any semistable torsion free sheaf on $X$ with rank $n$ and degree $d$. Then $E$ is spanned. Let a be the type of $E$ and set $u:=\max \{n+1, n+a\}$. Then for a general $u$-dimensional linear subspace of $H^{0}(C, E)$ the evaluation map $e_{E, V}$ is surjective.

Proof. We have $h^{0}(C, E)=d \geq 2 n \geq u$. The proof of Lemma 9 gives that $E$ is spanned outside $Q$. There are infinitely many degree 2 Cartier divisors $Z$ of $C$ such that $Z_{\text {red }}=\{Q\}$. Fix a general such $Z$. Since $\operatorname{deg}(E(-Z))=d-2 n \geq 0$ and $E(-Z)$ has no trivial factor even when $d=2 n$ by the generality of $Z$, $h^{1}(C, E(-Z))=0$. Hence the restriction map $H^{0}(C, E) \rightarrow H^{0}(C, E \mid Z)$ is surjective. Since $Z$ is zero-dimensional, the restriction map $H^{0}(C, E \mid Z) \rightarrow$ $H^{0}(C, E \mid\{Q\}$ is surjective. Hence $E$ is spanned at $Q$. Since $E$ is locally free outside $u$, the last assertion is a straightforward consequence of the spannedness of $E$ and of the inequality $h^{0}(C, E) \geq u$.

Now we give another proof of Lemma 13 without the assumption that the factors of $E$ are pairwise non-isomorphic.

Proposition 2. Fix integers $d>n>0$. Let $E$ be any polystable torsion free sheaf with, degree $d$ and rank $n$. Then $E$ is spanned. If $E$ has type $\leq 1$, then for a general $(n+1)$-dimensional linear subspace of $H^{0}(C, E)$ the evaluation map $e_{E, V}$ is surjective.

Proof. Lemma 9 shows that $E$ is spanned, except at $Q$. If $d \geq 2 n$, then use Lemma 14. Assume $n<d<2 n$. Hence $n \geq 2$. We also assume that the result is true for all pairs $\left(n^{\prime}, d^{\prime}\right)$ such that $d^{\prime}>n^{\prime}$ and $1 \leq n^{\prime}<n$. First assume $E$ stable. Hence $(n, d)=1$. By part (iii) of Theorem 1 there is an exact sequence

$$
0 \rightarrow E_{1} \rightarrow E \rightarrow E_{2} \rightarrow 0
$$

with $E_{1}$ and $E_{2}$ stables, $E_{1}$ locally free, $1 \leq \mu\left(E_{1}\right)<2$ and $1<\mu\left(E_{2}\right)<1$. Hence $h^{1}\left(C, E_{1}\right)=0$. Thus $E$ is spanned if $E_{1}$ and $E_{2}$ are spanned. The inductive assumption gives that $E_{2}$ is spanned and that $E_{1}$ is spanned, unless $\mu\left(E_{1}\right)=1$. Assume $\mu\left(E_{1}\right)=1$, i.e. assume $\operatorname{deg}\left(E_{1}\right)=\operatorname{rank}\left(E_{1}\right)$. Since $E_{1}$ is stable, we get $\operatorname{rank}\left(E_{1}\right)=\operatorname{deg}\left(E_{1}\right)=1$. Since $E_{1}$ is locally free, it has its only section does not vanishes at $Q$. Hence $E_{1}$ is spanned at $Q$. Since $h^{1}\left(C, E_{1}\right)=0$ and $E_{2}$ is spanned, $E$ is spanned at $Q$. Now assume $E$ polystable, but not stable, say $E=\oplus_{i=1}^{j} F_{j}$ with $s \geq 2, F_{j}$ stables and $\mu\left(F_{j}\right)=\mu(E)$ for all $E$. Hence $\mu\left(F_{j}\right)>1$. Since $F_{j}$ is stable and of rank $n / s<n, F_{j}$ is spanned. Hence $E=\oplus_{i=1}^{j} F_{j}$ is spanned. For the last assertion use that $E$ has type $\leq 1$.

Proposition 3. Fix integers $k, d$, such that $k>0$ and either $k<d$ or $k=$ $d$ and $(n, d)=1$. Fix a polystable torsion free sheaf $E$ with pairwise non isomorphic summands of rank $n$ and degree $d$. Then for a generic $V \subset H^{0}(E)$ the coherent system $(E, V)$ is $0^{+}$-stable. 
Proof. The proof is essentially the same of the smooth case ([11], Theorem 4.1). A coherent subsystem $(F, W)$ which violates the $0^{+}$-stability must have $\mu(F)=\mu(E)$ i.e. $F$ must be a sum of factors of $E$. In particular we have a finite number of such subsheaves (if $(n, d)=1$ we have nothing to prove). For those subsheaves we have to check

$$
\frac{\operatorname{dim}\left(H^{0}(F) \cap V\right)}{\operatorname{rank}(F)}<\frac{k}{n} .
$$

Since $V$ is generic we have for all subsheaves $F$ of slope $\mu(F)=\mu(E)$

$$
\frac{\operatorname{dim}\left(H^{0}(F) \cap V\right)}{\operatorname{rank}(F)}=\max \left\{0, h^{0}(F)-d+k\right\}
$$

If this number is 0 equation (13) holds trivially. So we assume $h^{0}(F)=\operatorname{deg} F>$ $d-k$; then equation (13) becomes

$$
\frac{\operatorname{deg} F-d+k}{\operatorname{rank}(F)}<\frac{d-d+k}{n},
$$

which holds for every proper subsheaf.

Lemma 15. Fix positive integers $m, k$ and a rank $m$ degree $k$ polystable torsion free sheaf $F$ on $C$. Let $E$ be the general extension of $F$ by $\mathcal{O}_{C}^{\oplus k}$. Then $E$ is torsion free, polystable and with the same type as $F$.

Proof. Obviously, $E$ is torsion free and with the same type as $F$. We have $h^{0}(C, F)=k$ and for a general extension the coboundary map $\delta: H^{0}(C, F) \rightarrow$ $H^{1}\left(C, \mathcal{O}_{C}^{\oplus k}\right)$ is an isomorphism. As in [1] we will say that an extension of $F$ by $\mathcal{O}_{C}^{\oplus k}$ us complete if the associated coboundary map $\delta$ is an isomorphism. Since $\operatorname{dim}\left(\operatorname{Ext}^{1}\left(F, \mathcal{O}_{C}^{\oplus k}\right)\right)=k^{2}$ the group $G L(k) \cong \operatorname{Aut}\left(\mathcal{O}_{C}^{\oplus k}\right)$ acts transitively on the set of all complete extension. Hence, up to isomorphisms, there is a unique such extension. $h^{1}(C, E)=0$ and $h^{0}(C, E)=k$ for any such $E$. Conversely, $F$ is uniquely determined from $E$, because $F \cong e_{E, H^{0}(C, E)}$. Hence any automorphism of $E$ induces an automorphism of $F$. To check the polystability of $E$ we will use induction on the integer $m+k$. First assume $m=1$, while $k$ is arbitrary. If $F$ is locally free (resp. $\left.F \cong F_{1, k}\right)$, then use that for any $E \in U(k+1, k)$ (resp. $E \in V(k+1, k)$ ) the evaluation map $e_{E, H^{0}(C, E)}$ is injective and it has torsion free cokernel. Now assume $m \geq 2$ and that the result is true for all integers $m^{\prime}, k^{\prime}$ such that $2 \leq m^{\prime}+k^{\prime}<m+k$. First assume $(m, k)=h>0$. Hence $F \cong \oplus_{i=1}^{h} F_{i}$ with $F_{i}$ polystable of degree $k / h$ and rank $m / h$. Apply the inductive assumption to the pair of integers $(m / h, k / h)$ and take $E:=\oplus_{i=1}^{h} E_{i}$ with $E_{i}$ extension of $F_{i}$ by $\mathbb{O}_{C}^{\oplus k / h}$. Now assume $(m, k)=1$, i.e. assume that $F$ is stable. Assume that $E$ is not stable, i.e. (since $(m+k, k)=1$ ) assume the existence of a proper saturated subsheaf $G$ of $E$ such that $\mu(E / G)>\mu(G)$. Thus $h^{0}(C, \operatorname{Hom}(E / G, G))$; 0 . Thus $E$ is not simple and it has an endomorphism $u: E \rightarrow E$ such that $u \neq 0, u(E) \subseteq E_{1}$ and $u^{2}=0$. Since $u$ induces an endomorphism $u_{*}: H^{0}(C, E) \rightarrow H^{0}(C, E)$, it induces an endomorphism $v: F \rightarrow F$. Since $u^{2}=0$ and $u^{2}$ induces $v^{2}, v^{2}=0$. Since $F$ is simple, $v=0$. 
Since $v=0, u(E) \subseteq \mathcal{O}_{C}^{\oplus k}$. However, since $h^{1}(C, E)=0$, there is a non-zero morphism $E \rightarrow \mathcal{O}_{C}$, contradiction.

Lemma 16. Fix integers $d, k, n$ such that $n>k>0$ and $d \geq k$. Let $E$ be a general element of $U(n, d)$ and $V$ a general $k$-dimensional linear subspace of $H^{0}(C, E)$. Then $e_{E, V}$ is injective and $\operatorname{Coker}\left(e_{E, V}\right) \in U(n-k, d)$.

Proof. It is sufficient to find just one pair $(E, V)$ for which the lemma is true. If $d=k$, use Lemma 15. Now assume $d>k$. There is $E^{\prime} \in U(n, k)$ and an inclusion $j: E^{\prime} \rightarrow E$ with $E^{\prime} \in U(n, k)$ (Lemma 7). Furthermore, we may assume that $E^{\prime}$ is general in $U(n, k)$ (Lemma 7). Set $N:=H^{0}\left(C, E^{\prime}\right)$ and use $j$ to see $M$ as a $k$-dimensional linear subspace of $H^{0}(C, E)$. For general $E$ we may see $j$ as the composition of $d-k$ general positive elementary transformations supported by general points of $C_{r e g}$. For general $(E, j), \operatorname{Im}\left(e_{E, M}\right)$ is saturated in $E$ and $\operatorname{Coker}\left(e_{E, M}\right)$ is obtained from $\operatorname{Coker}\left(e_{E^{\prime}, N}\right)$ making $d-k$ general positive elementary transformations supported by general points of $C_{\text {reg }}$. Since $\operatorname{Coker}\left(e_{E^{\prime}, N}\right)$ may be considered as a general element of $U(n-k, k)$, Lemma 7 gives the polystability of $\operatorname{Coker}\left(e_{E, M}\right)$.

Just quoting Lemma 8 instead of Lemma 7 in the proof of Lemma 16 we get the following result.

Lemma 17. Fix integers $d, k, n$ such that $n>k>0$ and $d \geq k$. Let $E$ be a general element of $V(n, d)$ and $V$ a general $k$-dimensional linear subspace of $H^{0}(C, E)$. Then $e_{E, V}$ is injective and $\operatorname{Coker}\left(e_{E, V}\right) \in V(n-k, d)$.

Remark 6. Fix real numbers $\alpha>\beta$. Let $(E, V)$ be a coherent system on $C$. If $(E, V)$ is $\alpha$-stable and $\beta$-stable, then it is $\gamma$-stable for all $\alpha>\gamma>\beta$. The same is true for semistablity.

Theorem 2. Fix integers $d, n, k$ such that $d \geq n+2$ and $n>k>0$. Fix a general $E \in V(n, d)$ and a general $k$-dimensional linear subspace $V$ of $H^{0}(C, E)$. Then the coherent system $(E, V)$ is $\alpha$-stable for all $0<\alpha<d /(n-k)$.

Proof of Theorem 2. By Remark 6 and Proposition 3 it is sufficient to prove the existence of $\epsilon>0$ such that for every $d /(n-k)-\epsilon<\alpha<d /(n-k)$ there in a $k$ dimensional linear subspace $M$ of $H^{0}(C, E)$ such that $(E, M)$ is $\alpha$-stable. By Proposition 1 and the generality of $E$ there is an exact sequence

$$
0 \rightarrow G \stackrel{j}{\rightarrow} E \rightarrow \mathbb{K}_{Q} \rightarrow 0
$$

where $G$ is a general element of $U(n, d-1)$. Let $M$ be a general $k$-dimensional linear subspace of $G$. By [2] or Lemma $16 \operatorname{Coker}\left(e_{G, M}\right)$ is a polystable vector bundle with pairwise non-isomorphic factors. Hence we have an exact sequence

$$
0 \rightarrow \mathcal{O}_{C}^{\oplus k} \rightarrow G \rightarrow F \rightarrow 0
$$

in which $F^{\prime}:=\operatorname{Coker}\left(e_{E, M}\right) \in V(n-k, d)$ (Lemma 17). The polystability of $F^{\prime}$ is sufficient to copy the proof of [11], Th. 4.2 . 
Proposition 4. Suppose $0<k<n$ and either $k<d$ or $k=d$ and $(n, d)=$ 1. The general $(E, V) \in U(\alpha, n, d, k) \cup V(\alpha, n, d, k)$ is $\alpha$-stable for all $\alpha \in$ $\left(0, \frac{d}{n-k}\right)$.

Proof. By Lemmas 16 and 17 the coherent system is associated to a sequence

$$
0 \rightarrow \mathcal{O}^{k} \stackrel{\phi}{\rightarrow} E \rightarrow G \rightarrow 0
$$

where $G$ is a polystable torsion free sheaf with non-isomorphic factors. Consider the coherent subsystem $(F, W)$ with $\operatorname{dim} W=k$ and $F=\phi(V \otimes \mathcal{O})$. We obtain

$$
0 \rightarrow(F, W) \rightarrow(E, V) \rightarrow(G, 0) \rightarrow 0
$$

Now $(F, W)$ and $(G, 0)$ are $\alpha$-semistable coherent systems for all $\alpha>0$ and $\mu_{\alpha}(F, W)=\mu_{\alpha}(G, 0)$ for $\alpha=\left(\frac{d}{n-k}\right)$. This proves the $\left(\frac{d}{n-k}\right)$-semistability of $(E, V)$, so the claim follows by proposition 3 and by the fact that the set $\{\alpha \mid(E, V)$ is $\alpha$-stable $\}$ is an open interval with a finite number of critical value.

Proposition 5. Fix positive integers $x, k, n$ such that $n \neq k$ and $n \equiv 0$ $(\bmod k)$. Let $E$ be the direct sum of $n$ rank 1 torsion free sheaves, all of them of degree $x$. Let $V$ be a general $k$-dimensional linear subspace of $H^{0}(C, E)$. Then the coherent system $(E, V)$ is $\alpha$-stable for all $0<\alpha<x n /(n-k)$.

Proof. Write $E=\oplus_{i=1}^{k} E_{i}$ with each $E_{i}$ sum of $n / k$ of the rank 1 factors of $E$. Take a non-zero section of each $E_{i}$ and let $N$ be $k$-dimensional linear subspace spanned by these $k$ section. The coherent system $(E, M)$ is $\alpha$-polystable for all $0<\alpha<x n /(n-k)$. Hence if the proposition fails there is an integer $m$ such that $k>m \geq 1, k \equiv 0(\bmod m)$ and a rank $n / m$ factor $G$ of $E$ such that $\operatorname{dim}\left(V \cap H^{0}(C, G)\right)=k / m$. Fix $m$. The set of all rank $n / m$ factors of $E$ is an irreducible variety $T_{m}$ of dimension $n / m(n-n / m)$. The set of all $k$-dimensional linear subspaces of $H^{0}(C, E)$ is an irreducible variety $\Gamma$ of dimension $k(n x-k)$. For each $G \in T_{m}$ let $B(G)$ be the set of all $W \in \Gamma$ such that $\operatorname{dim}\left(W \cap H^{0}(C, G)\right) \geq k / m . B(G)$ is an irreducible variety of dimension $k / m(x n / m-k / m)+(k-k / m)(x n-x n / m-k+k / m)$. Since $k>m, k(n x-k)>$ $n / m(n-n / m)+k / m(x n / m-k / m)+(k-k / m)(x n-x n / m-k+k / m)$, concluding the proof.

\section{Some OTHER CASES}

We start with the easiest case, i.e. when $\operatorname{dim}(V)=\operatorname{rk}(E)$.

Proposition 6. Fix integers $d>n>0$ and any $E \in U(n, d) \cup V(n, d)$. Let $V \subset H^{0}(C, E)$ be a general $n$-dimensional linear subspace. Then the coherent system $(E, V)$ is $\alpha$-stable for all $\alpha>0$.

Proof. The evaluation map $e_{E, V}$ is injective (Remark 5). The proof made in the smooth case ([5], Th. 5.6, or [11], Th. 5.4) works verbatim because it only uses the injectivity of $e_{E, V}$, that $E$ is polystable and that $E$ has only finitely many direct factors. 
Proposition 7. Fix integers $n>0$ and $x \geq 2$. Let $E$ be any direct sum of $n$ rank 1 torsion free sheaves, all of them with degree $x$. Let $V$ be a general $n$-dimensional linear subspace of $H^{0}(C, E)$. Then the coherent system $(E, V)$ is $\alpha$-stable for all $\alpha>0$.

Proof. By Remark 6 it is sufficient to show that $(E, V)$ is $0^{+}$-coherent and that it is $\alpha$-coherent for all $\alpha \gg 0$. it is sufficient to show that for every $\alpha>0$ there is an $n$-dimensional linear subspace $M$ of $H^{0}(C, E)$ such that $(E, M)$ is $\alpha$-stable. The evaluation map $e_{E, V}$ is injective (Remark 5). Write $E=\bigoplus_{i=1}^{n} L_{i}$ with $\operatorname{rank}\left(L_{i}\right)=1$ for all $i$. Fix $e_{i} \in H^{0}\left(C, L_{i}\right), e_{i} \neq 0$, and see $e_{i}$ as a section of $E$. Let $W$ be the $n$-dimensional linear subspace of $H^{0}(C, E)$ generated by $e_{1}, \ldots, e_{n}$. The coherent system $(E, W)$ is $\alpha$-polystable for all $\alpha$. Hence $(E, V)$ is $\alpha$-semistable and it is not $\alpha$-stable if and only if there is a proper direct factor $G$ of $E$ such that $\operatorname{dim}\left(V \cap H^{0}(C, G)\right)=\operatorname{rank}(G)$. Fix an integer $m$ such that $1 \leq$ $m \leq n-1$. $E$ has at most $m(n-m)$ different rank $m$ factors. Fix one of them, $A$. We have $h^{0}(X, A)=m x$. Let $G\left(n, H^{0}(C, E)\right)$ be the Grassmannian of all $n$-dimensional linear subspaces of $H^{0}(C, E) . G\left(n, H^{0}(C, E)\right)$ is an irreducible variety and $\operatorname{dim}\left(G\left(n, H^{0}(C, E)\right)\right)=n(n x-n)=n^{2}(x-1)$. Set $B(A):=\{M \in$ $\left.G\left(n, H^{0}(C, E)\right): \operatorname{dim}\left(M \cap H^{0}(C, A)\right) \geq m\right\} . B(A)$ is an irreducible variety of dimension $m(m x-m)+(n-m)((n-m) x-(n-m))=m^{2} x+(n-m)^{2} x-m^{2}-$ $(n-m)^{2}$ (a Schubert cycle). Since $m(n-m)+m^{2} x+(n-m)^{2} x-m^{2}-(n-m)^{2}<$ $n^{2}(x-1)$, a general $V \notin B(A)$ for any $A$ and any $m$.

In the next proposition we extend the technique of the smooth case the resolve the $k=n+1$ case.

Proposition 8. Fix integers $d \geq n+2>0$ and $E \in U(n, d) \cup V(n, d)$. Let $V \subseteq H^{0}(C, E)$ be a general linear subspace with dimension $n+1$. Then the coherent system $(E, V)$ is $\alpha$-stable for all $\alpha>0$.

Proof. Lemma 13 implies that the evaluation map $j_{V}: V \otimes \mathcal{O}_{C} \rightarrow E$ is surjective. Fix an integer $r$ such that $0<r<n$ and a rank $r$ subsheaf $F$ of $E$. Set $W:=H^{0}(C, F) \cap V$. Since $j_{V}$ is generically surjective and $r<n, W \neq V$.

Claim: The evaluation map $j_{W}: W \otimes \mathcal{O}_{C} \rightarrow E$ is injective.

Proof of the Claim: Since $W \neq V$, there is an $n$-dimensional linear subspace $M$ of $V$ such that $W \subseteq M$. It is sufficient to prove the injectivity of $j_{M}$. Assume that $j_{M}$ is not injective. Then $\operatorname{Coker}\left(j_{M}\right)$ is a coherent sheaf of rank at least 1 which is a quotient of $E$. Since $E$ is spanned by $V, \operatorname{Coker}\left(j_{M}\right)$ is spanned by $V / M$. Since $\operatorname{dim}(M / V)=1 \geq \operatorname{rank}\left(\operatorname{Coker}\left(j_{M}\right)\right)$, this implies $\operatorname{Coker}\left(j_{M}\right) \cong \mathcal{O}_{C}$. Since $E$ is spanned, the existence of a surjective map $E \rightarrow \mathcal{O}_{C}$ implies that $\mathcal{O}_{C}$ is a direct factor of $E$, contradicting the semistability of $E$.

Since the evaluation map $j_{W}: W \otimes \mathcal{O}_{C} \rightarrow E$ is injective, $F$ has rank $r$ and $\operatorname{Im}\left(j_{W}\right) \subseteq F$, we have $\operatorname{dim}(W) \leq r$. Thus $\mu_{\alpha}(F, W) \leq \mu(F)+\alpha<$ $\mu(E)+(1+1 / n) \alpha=\mu_{\alpha}(E)$ for all $\alpha>0$. Hence $(E, V)$ is $\alpha$-stable. 
We conclude giving examples of $\alpha$-stable coherent systems in the case when $\operatorname{dim}(V)=\operatorname{rk}(E)+2$.

Remark 7. Let $A$ be a rank $n$ polystable torsion free sheaf whose indecomposable factor are pairwise non-isomorphic. Fix an integer $m>0$ and $m$ distinct points $P_{1}, \ldots, P_{m} \in C_{r e g}$. Set $d:=\operatorname{deg}(A)$ and $I\left(P_{i}\right):=H^{0}\left(C, A\left(-P_{i}\right)\right.$, $1 \leq i \leq m$. Now we fix an integer $k$ such that $d \geq k>n$ and a general $k$-dimensional linear subspace $V$ of $H^{0}(C, A)$. Since $V$ is general, $\operatorname{dim}(V \cap$ $\left.I\left(P_{i}\right)\right)=\max \{0, k-n\}$ for all $i$. Fix an integer $r$ such that $2 \leq r \leq n$. Let $V(r)$ denote the Grassmannian of all $r$-dimensional linear subspace of $V$. Let $B(V, r)$ be the subset of $V(r)$ formed by all $M \in V(r)$ such that the evaluation map $j_{M}: M \otimes \mathcal{O}_{C} \rightarrow A$ is not injective. Set $B\left(V, r, P_{i}\right):=\{M \in V(r)$ : $\left.M \cap I\left(P_{i}\right) \neq\{0\}\right\}$. Notice that $B\left(V, r, P_{i}\right):=\left\{M \in V(r): M \cap I\left(P_{i}\right) \neq\{0\}\right.$ and $B(V, r) \subseteq \cap_{i=1}^{m} B\left(V, r, P_{i}\right)$.

First Claim: Assume that $\left(P_{1}, \ldots, P_{m}, V\right)$ is a general $(m+1)$-ple. Then $\operatorname{dim}\left(\left\langle\cup_{j=1}^{i}\left(V \cap I\left(P_{j}\right)\right)\right\rangle=\min \{k, m(k-n)\}\right.$.

Proof of the First Claim: Since $\operatorname{dim}\left(V \cap I\left(P_{j}\right)\right)=k-n$ and $\operatorname{dim}(V)=k$, the inequality $\operatorname{dim}\left(\left\langle\cup_{j=1}^{i}\left(V \cap I\left(P_{j}\right)\right)\right\rangle \geq \min \{k, m(k-n)\}\right.$. Since $\operatorname{dim}(V \cap$ $\left.I\left(P_{1}\right)\right)=k-n$, the First Claim is true. Assume $2 \leq i \leq m$ and that the Claim is true for the integer $i^{\prime}:=i-1$. Set $M:=\left\langle\cup_{j=1}^{i-1}\left(V \cap I\left(P_{j}\right)\right)\right\rangle$. The inductive assumption gives $\operatorname{dim}(M)=\min \{k,(i-1)(k-n)$. Since the First Claim is true for all integers $x \geq i$ if $\operatorname{dim}(M)=k$, we may assume $\operatorname{dim}(M)=(i-1)(k-n)$. Let $N_{j} \subset I\left(P_{j}\right), 1 \leq j \leq i$, be general linear subspaces such that $\operatorname{dim}\left(N_{j}\right)=$ $k-n$. The generality of all $N_{j}$ implies $\operatorname{dim}\left(\left\langle\cup_{j=1}^{i-1} N_{j}\right\rangle\right)=(i-1)(k-n)$ and $\operatorname{dim}\left(\left\langle\cup_{j=1}^{i} N_{j}\right\rangle\right)=\min \{k, i(k-n)\}$. We fix $P_{1}, ; P_{i}$, but we change $V$ : we take as $V$ a general linear subspace of $H^{0}(C, A)$ containing each $N_{j}, 1 \leq j \leq i$. For such vector space $V$ we have $N_{j}=I\left(P_{j}\right) \cap V$ for all $1 \leq j \leq i$. Hence the equality $\operatorname{dim}\left(\left\langle\cup_{j=1}^{i} N_{j}\right\rangle\right)=\min \{k, i(k-n)\}$ is equivalent to the truth of the Claim for the integer $i$. Inductively we get the First Claim.

Now we fix integers $d \geq k>n \geq r \geq 2$ and take $m:=r+1$. Assume $k \geq(r+1)(k-n)$, i.e. assume $n<k \leq(r+1) n / r$. Fix a general $(m+1)$-ple $\left(P_{1}, \ldots, P_{m}, V\right)$.

Second Claim: For every $W \in V(r)$ the evaluation map is injective.

Proof of the Second Claim: Fix any $W \in V(r)$ and assume $W \in B(V, r)$. Hence $W \in \cap B\left(V, r, P_{i}\right)$ for all $i=1, \ldots, r+1$. Hence $W \cap I\left(P_{i}\right) \cap V \neq\{0\}$. The First Claim and the assumption $k \geq(r+1)(k-n)$ imply that the $r+1$ linear spaces $I\left(P_{i}\right) \cap V, 1 \leq i \leq r+1$, are linearly independent. Hence the $r+1$ non-zero linear spaces $W \cap I\left(P_{i}\right) \cap V, 1 \leq i \leq r+1$, cannot be contained in the $r$-dimensional linear space $W$. The contradiction proves the Second Claim.

Theorem 3. Fix integers $n, r, k$, d such that $2 \leq r \leq n<k \leq(r+1) n / r$ and $d \geq k$. Let $A$ be a rank $n$ polystable torsion free sheaf on $C$ such that $\operatorname{deg}(A)=d$ and its indecomposable factors are pairwise non-isomorphic. Let $V$ be a general $k$-dimensional linear subspace of $H^{0}(C, A)$.

(a) The evaluation map $j_{V}$ is surjective. 
(b) For every integer $r$ such that $2 \leq r \leq n$ and every $r$-dimensional linear subspace $M$ of $V$ the evaluation map $j_{M}$ is injective.

(c) Assume $k=n+2, n \geq 4$ and either $(d, n)=1$ or $d>k$. Then the coherent system $(A, V)$ is $\alpha$-stable for all $\alpha>0$.

Proof. Part (a) is Proposition 2. Part (b) is the Second Claim of Remark 7. Make all the assumptions of part (c) and fix $\alpha>0$, an integer $s$ such that $1 \leq s \leq n-1$ and a rank $s$ subsheaf $F$ of $A$. Set $W:=V \cap H^{0}(C, F)$ and $r:=\operatorname{dim}(W)$. Since $k>n$ and $A$ is semistable, we have $\mu_{\alpha}(F, W)<\mu_{\alpha}(A, V)$ if $r \leq s$. Now assume $r \geq s+2$. Since $V$ spans $A$, the $(n+2-r)$-dimensional vector space $V / W$ spans the coherent sheaf $A / F$. Since $A / F$ has generic rank $n-s$, this is possible only if $r=s+2$ and $A / F \cong \mathcal{O}_{C}^{\oplus(n-s)}$. Since $A$ is

spanned, the existence of a surjection $A \rightarrow \mathcal{O}_{C}^{\oplus(n-s)}$ implies that $\mathcal{O}_{C}^{\oplus(n-s)}$ is a direct factor of $A$, contradicting the semistability of $A$. Now assume $r=s+1$. We get $\mu_{\alpha}(A ; V)>\mu(F, W)$ if $2 s \geq n+1$. If $2 s=n$, we need also to use that $A \in U(n, d) \cup V(n, d)$ and that $V \neq H^{0}(C, A)$, since $d>n$. Hence we may assume $2 s<n$. Since $n \geq 4, r:=s+1 \leq n$. The Second Claim of Remark 7 gives the injectivity of $j_{W}$. Since $W \subseteq H^{0}(C, F)$, this implies $s \geq r$, contradiction.

\section{REFERENCES}

[1] M. F. Atiyah, Vector bundles over an elliptic curve, Proc. London Math. Soc. 7 (1957) $414-452$.

[2] E. Ballico, Holomorphic triples on singular genus one curves, Int. Jour. Contemp. Math. Sciences 2 (2007), no. 17, 847-850.

[3] U. N. Bhosle, Maximal subsheaves of torsion-free sheaves on nodal curves, J. London Math. Soc. (2) 74 (2006), no. 1, 59-74.

[4] L. Bodnarchuck, I. Burban, Y. Drozd, G.-M. Greuel, Vector bundles and torsion free sheaves on degenerations of elliptic curves. In Global aspects of complex geometry, pages 83-128. Springer, Berlin, 2006.

[5] S. B. Bradlow, O. García-Prada, V. Muñoz and P. E. Newstead, Coherent systems and Brill-Noether theory, Internat. J. Math. 14 (2003), no. 7, 683-733.

[6] P. R. Cook, Local and global aspects of the module theory of singular curves, Ph. D. thesis, Liverpool, 1993.

[7] G.-M. Greuel and H. Knörrer, Einfache Kurvensingularitäten und torsionfreie Moduln, Math. Ann. 270 (1985), 417-425.

[8] R. Friedman, J. W. Morgan and E. Witten, Vector bundles over elliptic fibrations, J. Algebraic Geom. 8 (1999), no. 2, 279-401.

[9] A. Hirschowitz, Problème de Brill-Noether en rang supérieur, Preprint 91, Université de Nice, Nice, 1985.

[10] A. King and P. E. Newstead, Moduli of Brill-Noether pairs on algebraic curves, Internat. J. Math. 6 (1995), no. 5, 733-748.

[11] H. Lange and P. E. Newstead, Coherent systems on elliptic curves, Internat. J. Math. 16 (2005), no. 7, 787-805.

[12] S. Mozgovoy, Classification of semistable sheaves on a rational curve with a node, arXiv:math/AG:0410190.

[13] F. Prantil and S. Pasotti, Holomorphic triples on elliptic curves, Results in Math. (to appear). 
[14] T. Teodorescu, Semistable torsion-free sheaves over curves of arithmetic genus one, $\mathrm{Ph}$. D. thesis, Columbia University, 1999.

\section{Received: June 12, 2007}

